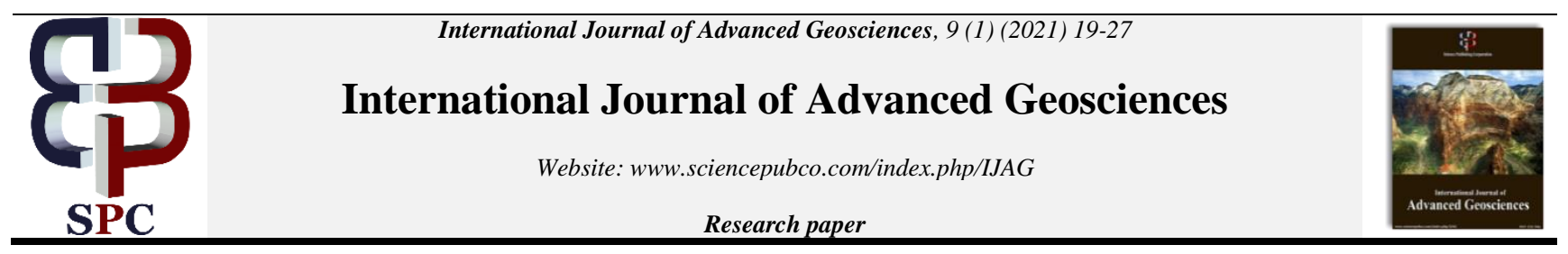

\title{
Factor analyses of transformed geochemical compositional data of meme river stream sediment, Lokoja, north central Nigeria
}

\author{
Ngozi-Chika C. S. ${ }^{1,2}$, Olorunyomi, A. E. ${ }^{3}$, Echetema H. N. ${ }^{4}$, Ibrahim O. I. ${ }^{5}$ \\ ${ }^{1}$ Department of Earth Sciences, Salem University, P.M.B. 1060, Lokoja, Nigeria \\ ${ }^{2}$ Department of Geology and Mineral Sciences, University of Ilorin, P.M.B. 1515, Ilorin Nigeria \\ ${ }^{3}$ Department Of Geology, University of Jos, Jos, P.M.B, 2084, Nigeria \\ ${ }^{4}$ Department Of Geology, Federal University of Technology, Owerri, Nigeria \\ ${ }^{5}$ Lower Niger River Basin Development Authority, P.M.B. 1529, Ilorin, Nigeria \\ *Corresponding authorE-mail: ngozichikastephen@yahoo.com
}

\begin{abstract}
Geochemical mapping using stream sediments from MRDB, north-central, Nigeria was undertaken towards obtaining multivariate association patterns reflecting the presence of ore mineralization in Lokoja region. The area is underlain by Precambrian crystalline rocks within the Benin-Nigeria Shield and clastic sedimentary rocks of Bida Basin (one of Nigeria inland sedimentary basins). The basement crystalline rocks have been known as a source of ore minerals in Nigeria. The major lithological units are cut by the Meme river watershed which have deposited in their tributaries, large quantities of alluvial and eluvial deposits formed during an extensive period of weathering and surficial processes. The PC analysis was performed on clr-transformed of Meme sediment geochemical compositional data of selected ore forming elements in the hope of obtaining geochemical information that could elucidate on the inferred ore mineralization of the region. The eight PCs explain about $93 \%$ of the total variance. The positive and negative loadings of PCs indicated the presences of oxides, sulphides, REEs and gems mineralisation in the region. Further interrogation of Spearman correlation of ilr transformed data with respect to the PC loadings indicated well developed relationship between $\mathrm{Sr}$ and $\mathrm{V}(0.55), \mathrm{Mn}$ and $\mathrm{Pb}(0.89), \mathrm{Mn}$ and $\mathrm{Ta}(0.77), \mathrm{Mn}$ and $\mathrm{Nb}(0.78)$, $\mathrm{Nb}$ vs $\mathrm{Ta}(0.98), \mathrm{Rb}$ and $\mathrm{Cr}(0.59)$, In and $\mathrm{As}$ (0.64), $\mathrm{Pb}$ and $\mathrm{Ga}(0.78), \mathrm{Sb}$ and $\mathrm{Au}(0.52), \mathrm{Ba}$ and $\mathrm{Cr}(0.50)$. The elemental association suggests that they are either indicator of their own mineralization or are suitable pathfinders to pertinent minerals in Lokoja region. The negative correlation between Fe with other ore elements indicated that the Fe is from both proximal and dextral sources probably due to many Fe formations and mineralisation (goethite, haematite \pm siderite - bearing sedimentary ironstone formations in the region). The high Spearman correlation coefficients between $\mathrm{Mn}, \mathrm{Nb}$ and Ta inferred that these ore elements are from the proximal sources because they are reliable pathfinders to pertinent oxides mineralisation in the region. Inferred proximal mineralisation in the region include beryl, topaz, columbite, quartzofeldspathic and quartz veins with anomalous concentration of Au as well as industrial minerals which are artisanally mined in places for industrial purposes.
\end{abstract}

Keywords: Meme River Watershed; Stream Sediment; Compositional Data; Logratio; PCA and Mineralisation.

\section{Introduction}

Delineating target exploration areas from stream sediment geochemical data is one of the most fundamental tasks in regional-scale geochemical investigations (Ghezelbash et.al., 2019; Carranza, 2011). That is because the compositions of stream sediments in each location represent materials (regolith, bedrock, mineralisation) upstream of or within a catchment basin (Ghezelbash et. al., 2019). The regional geochemical study using stream sediment by Lapworth et. al. (2012) not only provided geochemical informations but provided a rapid and practical approach for regional geochemical mapping in West Africa and plays a frontal role in geochemical mapping towards identification of target areas and various kinds of ore deposits for detailed explorations.

The geochemical mapping of Meme streams was undertaken towards the regional geochemical mapping of Lokoja and environs (Fig. 2). Meme River drains over a very large watershed from where sediments are eroded and discharged into it and a major tributary to both the Niger-Benue Rivers (Fig. 2). Thus, stream sediments from Meme river would reflect the chemical elements distribution of the watershed (Ngozi-Chika, et. al., in press). Thus, such sediments (from Meme River) would reflect the chemical elements distribution of the watershed area. The watershed is a second order drainage channel with network of local drainage channels which have deposited in their channels, large quantities of alluvial and eluvial deposits formed during an extensive period of weathering and surficial processes (Fig. 2). The watershed traverses across the basement and sedimentary terrains of major lithological units and tectonic histories and good prospect for ore formation (Figs.1 and 2). 
There are a number of important processes that explain the majority of the data variability in stream-sediment geochemistry. These include source geology and mineralization, physical hydromorphic processes (transport and winnowing), chemical processes (e.g., the important effects of Fe-Mn-oxyhydroxide and Fe-oxides on ore element distributions), weathering and erosion processes (Lapworth et.al., 2012). In this study, the resulting geochemical data of Meme stream sediment were subjected to multivariate analysis following an appropriate mathematical log-ratio transformation to eliminate closure problem associated with compositional data. This is to adequately interrogate the geological and geochemical processes, dispersion, anomaly and elemental associations which serve as ore elements indicator pertinent to ore mineralisation in the region.

Geochemical data are usually reported as parts of a total composition (ppm, $\mathrm{mg} / \mathrm{kg}$, percentages, etc.) and thus, affected by the closure problem (Sadeghi et.al., 2014; Reimann et al., 2008, 2012; Grunsky et al., 2014). Thus, working with the original scale of concentrations $(\mathrm{mg} / \mathrm{kg}$, percentages, etc.) leads to biased result. Stream sediment data are compositional and should be open prior to any statistical analysis using an appropriate mathematical logratio transformation method from a variety of suggested methods given by Aitchison (1986); Filzmoser et. al. (2009); Reimann et al. (2008); Carranza, (2011) and Egozcue et al. (2003). Various possibilities for data transformation of compositional data have been introduced in the literature (Filzmoser et. al., 2009); the most widely used is the family of one-to-one logratio transformations (Aitchison, 1986). Three different methods are used to open the compositional data in this family, namely: (1) an additive logratio (alr)-transformation (Aitchison, 1986); (2) a centered logratio (clr)-transformation (Aitchison, 1986); (3) an isometric logratio (ilr)-transformation (Egozcue et al., 2003). The studies by Carranza (2011) and Sadeghi et al. (2014) has shown that either clr or ilr-transformed compositional data are superior to alr-transformed compositional data for recognizing anomalous multi-element signatures associated with ore mineralization.

\section{The study area}

The study area is located in Lokoja, north-central Nigeria given in Fig. 1, is situated within the mineralized belt of the Western Province of the Nigerian Basement Complex (Precambrian) (Haruna, 2017; Fig. 3). The area is underlain by Precambrian crystalline rocks within the Benin-Nigeria Shield (of the Dahomeyan Terrane) that separates the Archaean to Mesoproterozoic West African and Congo Cratons to the west and east of Nigeria, respectively (Lapworth et al., 2014) and clastic sedimentary rocks of Bida Basin (one of Nigeria inland sedimentary basins in places). The basement rocks are believed to be a result of at least four major orogenic cycles of deformation, metamorphism and remobilization corresponding to the Liberian (2,700 Ma), the Eburnean (2,000 Ma), the Kibaran (1,100 Ma), and the Pan-African cycles (600 Ma) (Obaje et al., 2015). The Precambrian crystalline rocks comprises migmatites, gneisses, quartzites, granites and pegmatites with compositions variably including quartz, alkaline feldspars, muscovite, biotite and ferromagnesian minerals, notably pyroxenes occurring with anorthite, reported by Ngozi-chika et al. (in press). The basement crytalline rocks have been a known source of mineralisation in Nigeria. The sedimentary rocks are dominantly conglomerates, arkosic sandstones, siltstones, kaolinitic claystones and goethitic ironstones.

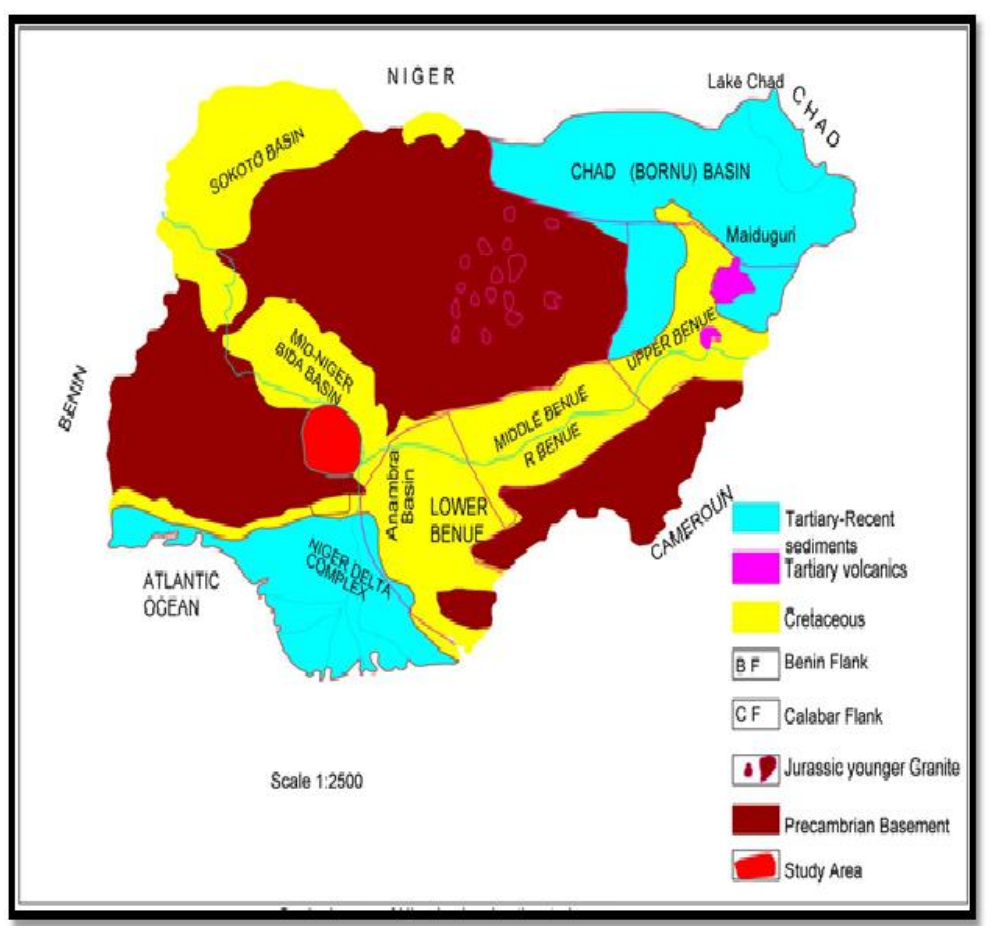

Fig. 1: Simplified Regional Geology Map of Nigeria and Location of the Study Area. 


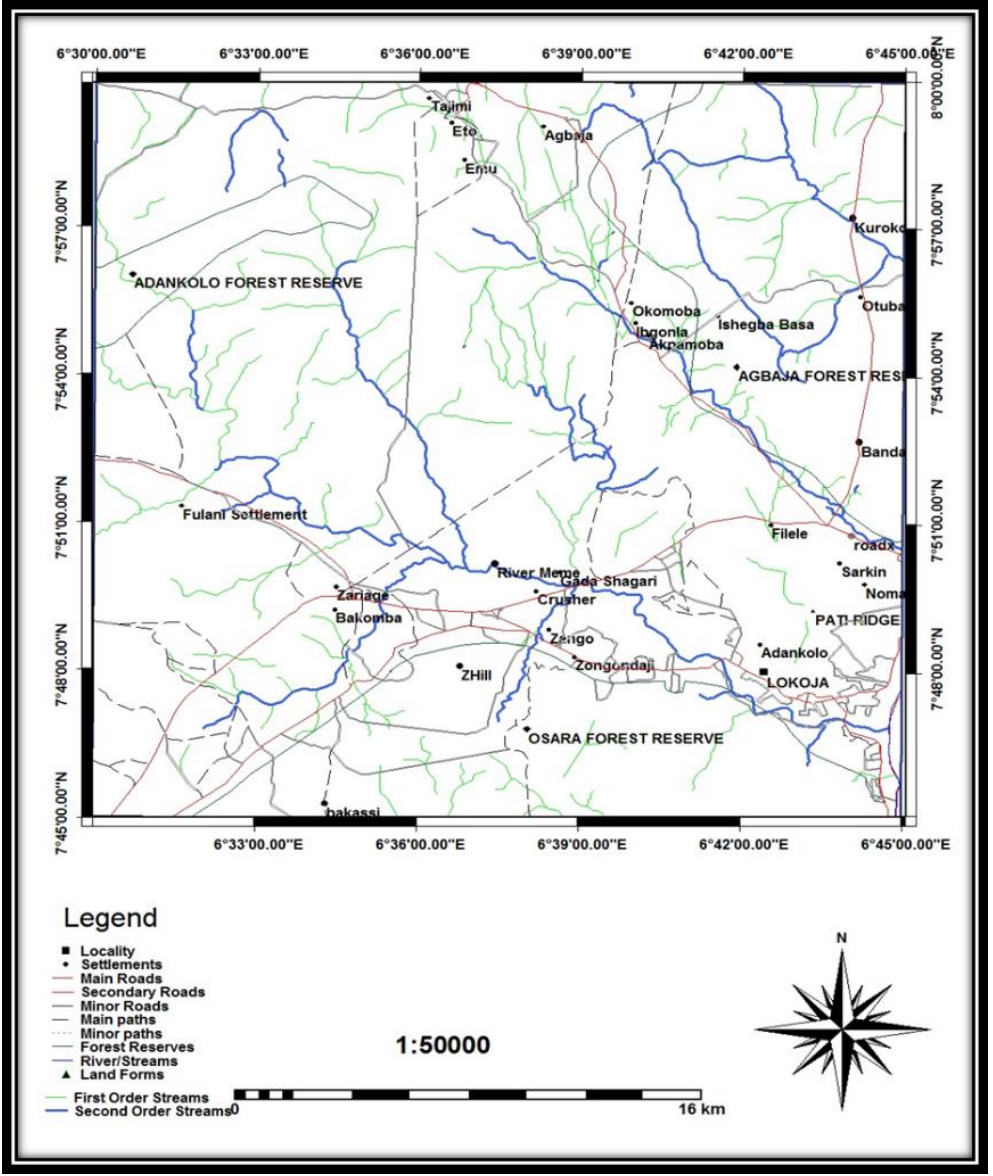

Fig, 2: Drainage of Map of Lokoja and Environs Showing First and Second Order Stream Chanels.

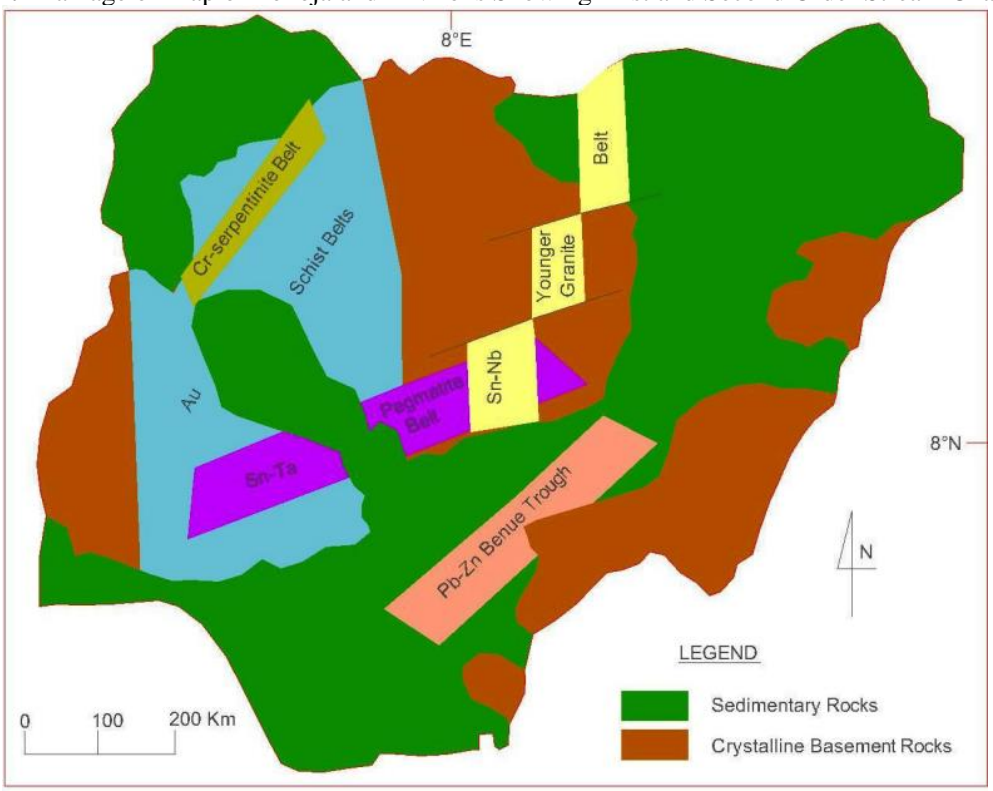

Fig. 3: Major Mineral Belts in Nigeria (Source: Haruna, 2017).

\section{Meme river drainage basin geochemical compositional data}

\subsection{Stream sediment geochemical sampling}

Geochemical exploration study was undertaken towards elucidating on the inferred mineralisation in parts of Lokoja and environs. Stream sediment samples were collected from various locations within the Meme River Drainage Basins (MRDB) (Ngozi-Chika et al. in press) with the hope of obtaining geochemical information that could elucidate on the indicated mineralization of the Lokoja region. Meme River is a second order streams that cut through the Precambrian crystalline rocks and the clastic sedimentary rocks. It is a major tributary to both the Niger-Benue Rivers and drains over a very large watershed from where sediments are eroded and discharged into it. Thus, such sediments (from Meme River) would reflect the chemical elements distribution of the watershed area. 


\subsection{Data processing methodology}

\subsubsection{Handling censored values and data transformations}

The censored values were handled following the method described and documented by Sadeghi et.al. (2014), Lapworth et.al. (2012) and Carranza (2011). Censored values were first examined and replaced by values equal to half of their respective detecting limits (DLs) as it is a customary procedure in geochemistry (Sadeghi et al., 2014).

The data transformations is require to open the compositional data for statistical and computational analysis (Sadeghi et al., 2014, Grunsky et al., 2014, Reimann et al., 2008 and Carranza, 2011). Three different methods are possible, namely: (1) an additive logratio (alr)-transformation (Aitchison, 1986); (2) a centered logratio (clr)-transformation (Aitchison, 1986); (3) an isometric logratio (ilr)-transformation (Egozcue et al., 2003). In this study, the clr-transformation is applied to perform the statistical PC analysis of compositional data as ilrtransformation will require back-transformation to clr-space for ease interpretation. Furthermore, Sadeghi et. al. (2014) has shown that clr transformed geochemical data provide enhancement of multivariate association patterns reflecting the presence of gold mineralisation in the Giyani Greenstone Belt (GGB), as compared to ln-transformed geochemical data. The authors' conclusion was similar to other real documentation and research on compositional data approach

\subsubsection{Transformations of meme stream sediment compositional data for multivariate analysis.}

Among the various multivariate statistical techniques for revealing patterns attributed to geological and mineralization processes. Principal Component analysis (PCA) has been employed for studying geochemical data structures (Sadeghi et. al., 2014 and Grunsky, 2010). For mapping of anomalies representing a multi-element signature of mineralization, log-ratio transformed data sets should be used as recommended by Carranza (2011) and Sadeghi et. al. (2014). Employing the steps reported in Sadeghi et. al. (2014), the clr-transformation was applied to perform the multivariate analysis for the ore elements as displayed in (Table 1). The choice of clr over ilr for multivariate analysis arise from the fact that ilr-transformation requires back-transformation to clr-space for ease interpretation and in-addition, clr- transformation have shown to give enhancement of multivariate elemental association patterns reflecting the presence of ore mineralization (Sadeghi et. al., 2014, Grunsky et al., 2014, Reimann et al., 2008 and Carranza, 2011). In this study, Equation (1) was employed for the clr-transformation prior to the multivariate statistical analysis.

$$
\operatorname{clr}(\mathbf{x})=\left(y_{1}, \ldots, y_{D}\right)=\left(\ln \frac{x_{i}}{\sqrt[D]{\prod_{i=1}^{D} x_{i}}}, \ldots, \ln \frac{x_{D}}{\sqrt[D]{\prod_{i=1}^{D} x_{i}}}\right) .
$$

\section{Results, interpretation, and discussion}

Summary of Meme stream sediments clr-transformed geochemical composition is given in Table 1, the Cluster dendogram analysis is given in Table 2 and Fig. 4 while principal component analyses is displayed in Tables 3, 4, 5, 6 and 7.

Table 1: Summary of Meme Stream Sediments Transformed Geochemical Composition

\begin{tabular}{lll} 
& Table 1: Summary of Meme Stream Sediments Transformed Geochemical Composition \\
\hline Element & Min. & Max. \\
\hline clr.V & -1.959 & 4.537 \\
clr.Cr & 1.337 & 4.276 \\
clr.Ni & -8.875 & 2.418 \\
clr.Cu & 0.609 & 3.941 \\
clr.Zn & 1.222 & 3.557 \\
clr.Ta & -9.346 & -1.071 \\
clr.Ga & -9.065 & 1.774 \\
clr.AS & -7.669 & 2.308 \\
clr.Pb & -0.992 & 2.530 \\
clr.Rb & 0.150 & 2.573 \\
clr.Sr & 1.260 & 4.277 \\
clr.Hg & -10.476 & 2.919 \\
clr.Zr & 2.724 & 8.764 \\
clr.Nb & -9.346 & -1.035 \\
clr.Mo & -10.476 & 1.835 \\
clr.Fe & 7.358 & 11.158 \\
clr.Au & -9.453 & 2.678 \\
clr.Ag & -2.152 & 2.290 \\
clr.Mn & 2.246 & 4.821 \\
clr.Re & -9.346 & 3.019 \\
clr.Eu & -2.841 & 5.153 \\
clr.Ba & 1.953 & 6.337 \\
clr.Ir & -9.453 & 0.965 \\
clr.In & -9.187 & 4.489 \\
clr.Ce & -9.346 & 2.707 \\
clr.Sb & -2.746 & 6.393 \\
clr.Y & -9.049 & 4.182 \\
\hline
\end{tabular}

Table 2: Stage and Clusters of Meme Stream Sediments Transformed Geochemical Composition

\begin{tabular}{|c|c|c|c|c|c|c|}
\hline \multirow{2}{*}{ Stage } & \multicolumn{2}{|c|}{ Cluster Combined } & \multirow{2}{*}{$\begin{array}{l}\text { Coefficients } \\
\text { Cluster } 1\end{array}$} & \multicolumn{2}{|c|}{ Stage Cluster First Appears } & \multirow{2}{*}{$\frac{\text { Next Stage }}{\text { Cluster } 2}$} \\
\hline & Cluster 1 & Cluster 2 & & Cluster 2 & Cluster 1 & \\
\hline 1 & 6.773 & 25.086 & 25.086 & 6.773 & 25.086 & 25.086 \\
\hline 2 & 4.551 & 16.857 & 41.943 & 4.551 & 16.857 & 41.943 \\
\hline 3 & 4.017 & 14.877 & 56.819 & 4.017 & 14.877 & 56.819 \\
\hline 4 & 2.555 & 9.464 & 66.284 & 2.555 & 9.464 & 66.284 \\
\hline
\end{tabular}




\begin{tabular}{|c|c|c|c|c|c|c|}
\hline 5 & 2.234 & 8.275 & 74.559 & 2.234 & 8.275 & 74.559 \\
\hline 6 & 1.968 & 7.289 & 81.848 & 1.968 & 7.289 & 81.848 \\
\hline 7 & 1.866 & 6.912 & 88.761 & 1.866 & 6.912 & 88.761 \\
\hline 8 & 1.261 & 4.669 & 93.430 & 1.261 & 4.669 & 93.430 \\
\hline 9 & .807 & 2.987 & 96.417 & & & \\
\hline 10 & .586 & 2.169 & 98.586 & & & \\
\hline 11 & .382 & 1.414 & 100.000 & & & \\
\hline 12 & $4.55 \mathrm{E}-016$ & $1.69 \mathrm{E}-015$ & 100.000 & & & \\
\hline 13 & $3.48 \mathrm{E}-016$ & $1.29 \mathrm{E}-015$ & 100.000 & & & \\
\hline 14 & $2.53 \mathrm{E}-016$ & $9.36 \mathrm{E}-016$ & 100.000 & & & \\
\hline 15 & $1.80 \mathrm{E}-016$ & $6.66 \mathrm{E}-016$ & 100.000 & & & \\
\hline 16 & $1.50 \mathrm{E}-016$ & $5.55 \mathrm{E}-016$ & 100.000 & & & \\
\hline 17 & $1.27 \mathrm{E}-016$ & $4.69 \mathrm{E}-016$ & 100.000 & & & \\
\hline 18 & $9.07 \mathrm{E}-017$ & $3.36 \mathrm{E}-016$ & 100.000 & & & \\
\hline 19 & $5.13 \mathrm{E}-017$ & $1.90 \mathrm{E}-016$ & 100.000 & & & \\
\hline 20 & $1.37 \mathrm{E}-017$ & $5.08 \mathrm{E}-017$ & 100.000 & & & \\
\hline 21 & $-4.89 \mathrm{E}-017$ & $-1.81 \mathrm{E}-016$ & 100.000 & & & \\
\hline 22 & $-1.22 \mathrm{E}-016$ & $-4.51 \mathrm{E}-016$ & 100.000 & & & \\
\hline 23 & $-1.39 \mathrm{E}-016$ & $-5.16 \mathrm{E}-016$ & 100.000 & & & \\
\hline 24 & $-1.90 \mathrm{E}-016$ & $-7.02 \mathrm{E}-016$ & 100.000 & & & \\
\hline 25 & $-2.17 \mathrm{E}-016$ & $-8.03 E-016$ & 100.000 & & & \\
\hline 26 & $-3.73 E-016$ & $-1.38 \mathrm{E}-015$ & 100.000 & & & \\
\hline 27 & $-3.83 \mathrm{E}-016$ & $-1.42 \mathrm{E}-015$ & 100.000 & & & \\
\hline
\end{tabular}

Rescaled Distance Cluster Combine

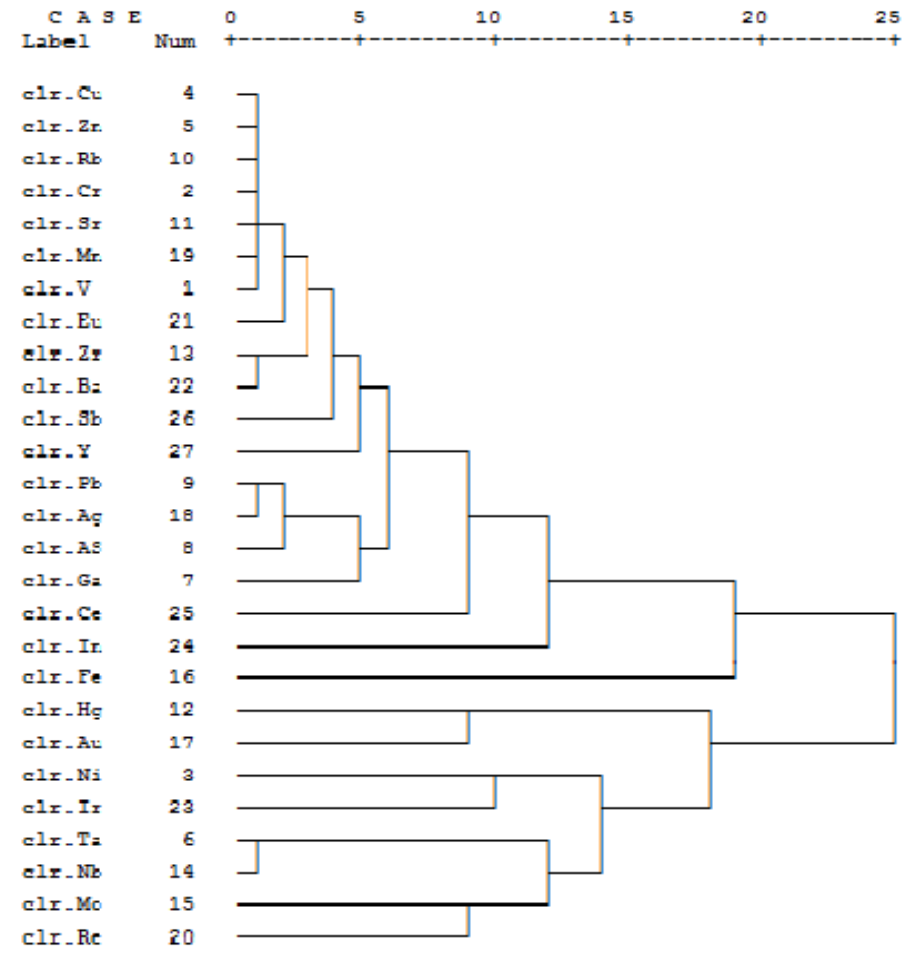

Fig. 4: Cluster Analysis Dendogram of Meme Stream Sediments Transformed Geochemical Composition.

Table 3: Kaiser-Meyer-Oklin (KMO) Measure of Sampling Adequacy (Communalities)

\begin{tabular}{lcc}
\hline Elements & Initial & Extraction \\
\hline clr.V & 1.000 & .906 \\
clr.Cr & 1.000 & .962 \\
clr.Ni & 1.000 & .942 \\
clr.Cu & 1.000 & .999 \\
clr.Zn & 1.000 & .818 \\
clr.Ta & 1.000 & .945 \\
clr.Ga & 1.000 & .953 \\
clr.AS & 1.000 & .982 \\
clr.Pb & 1.000 & .990 \\
clr.Rb & 1.000 & .924 \\
clr.Sr & 1.000 & .926 \\
clr.Hg & 1.000 & .956 \\
clr.Zr & 1.000 & .800 \\
clr.Nb & 1.000 & .961 \\
clr.Mo & 1.000 & .973 \\
clr.Fe & 1.000 & .994 \\
clr.Au & 1.000 & .927 \\
clr.Ag & 1.000 & .954 \\
clr.Mn & 1.000 & .931 \\
\hline
\end{tabular}




\begin{tabular}{lll}
\hline clr.Re & 1.000 & .988 \\
clr.Eu & 1.000 & .860 \\
clr.Ba & 1.000 & .993 \\
clr.Ir & 1.000 & .839 \\
clr.In & 1.000 & .988 \\
clr.Ce & 1.000 & .927 \\
clr.Sb & 1.000 & .919 \\
clr.Y & 1.000 & .868 \\
\hline
\end{tabular}

Table 4: Total Variance Meme Stream Sediment Transformed Geochemical Composition Explained

\begin{tabular}{|c|c|c|c|c|c|c|c|c|c|}
\hline \multirow{2}{*}{$\begin{array}{l}\text { Compo- } \\
\text { nent }\end{array}$} & \multicolumn{3}{|c|}{ Initial Eigenvalues } & \multicolumn{3}{|c|}{ Extraction Sums of Squared Loadings } & \multicolumn{3}{|c|}{ Rotation Sums of Squared Loadings } \\
\hline & Total & $\begin{array}{l}\% \text { of Vari- } \\
\text { ance }\end{array}$ & $\begin{array}{l}\text { Cumula- } \\
\text { tive } \%\end{array}$ & Total & $\begin{array}{l}\% \text { of Vari- } \\
\text { ance }\end{array}$ & $\begin{array}{l}\text { Cumula- } \\
\text { tive } \%\end{array}$ & Total & $\begin{array}{l}\% \text { of Vari- } \\
\text { ance }\end{array}$ & $\begin{array}{l}\text { Cumula- } \\
\text { tive } \%\end{array}$ \\
\hline 1 & 6.773 & 25.086 & 25.086 & 6.773 & 25.086 & 25.086 & 5.867 & 21.728 & 21.728 \\
\hline 2 & 4.551 & 16.857 & 41.943 & 4.551 & 16.857 & 41.943 & 3.345 & 12.390 & 34.119 \\
\hline 3 & 4.017 & 14.877 & 56.819 & 4.017 & 14.877 & 56.819 & 3.077 & 11.395 & 45.514 \\
\hline 4 & 2.555 & 9.464 & 66.284 & 2.555 & 9.464 & 66.284 & 2.787 & 10.323 & 55.837 \\
\hline 5 & 2.234 & 8.275 & 74.559 & 2.234 & 8.275 & 74.559 & 2.753 & 10.198 & 66.035 \\
\hline 6 & 1.968 & 7.289 & 81.848 & 1.968 & 7.289 & 81.848 & 2.729 & 10.109 & 76.144 \\
\hline 7 & 1.866 & 6.912 & 88.761 & 1.866 & 6.912 & 88.761 & 2.704 & 10.016 & 86.160 \\
\hline 8 & 1.261 & 4.669 & 93.430 & 1.261 & 4.669 & 93.430 & 1.963 & 7.270 & 93.430 \\
\hline 9 & .807 & 2.987 & 96.417 & & & & & & \\
\hline 10 & .586 & 2.169 & 98.586 & & & & & & \\
\hline 11 & .382 & 1.414 & 100.000 & & & & & & \\
\hline 12 & 4.55E-016 & $1.69 \mathrm{E}-015$ & 100.000 & & & & & & \\
\hline 13 & $3.48 \mathrm{E}-016$ & $1.29 \mathrm{E}-015$ & 100.000 & & & & & & \\
\hline 14 & $2.53 \mathrm{E}-016$ & $9.36 \mathrm{E}-016$ & 100.000 & & & & & & \\
\hline 15 & $1.80 \mathrm{E}-016$ & $6.66 \mathrm{E}-016$ & 100.000 & & & & & & \\
\hline 16 & $1.50 \mathrm{E}-016$ & $5.55 \mathrm{E}-016$ & 100.000 & & & & & & \\
\hline 17 & $1.27 \mathrm{E}-016$ & 4.69E-016 & 100.000 & & & & & & \\
\hline 18 & $9.07 \mathrm{E}-017$ & $3.36 \mathrm{E}-016$ & 100.000 & & & & & & \\
\hline 19 & $5.13 \mathrm{E}-017$ & $1.90 \mathrm{E}-016$ & 100.000 & & & & & & \\
\hline 20 & $1.37 \mathrm{E}-017$ & $5.08 \mathrm{E}-017$ & 100.000 & & & & & & \\
\hline 21 & $-4.89 \mathrm{E}-017$ & $-1.81 \mathrm{E}-016$ & 100.000 & & & & & & \\
\hline 22 & $-1.22 \mathrm{E}-016$ & $-4.51 \mathrm{E}-016$ & 100.000 & & & & & & \\
\hline 23 & $-1.39 \mathrm{E}-016$ & $-5.16 \mathrm{E}-016$ & 100.000 & & & & & & \\
\hline 24 & $-1.90 \mathrm{E}-016$ & $-7.02 \mathrm{E}-016$ & 100.000 & & & & & & \\
\hline 25 & $-2.17 \mathrm{E}-016$ & $-8.03 \mathrm{E}-016$ & 100.000 & & & & & & \\
\hline 26 & $-3.73 E-016$ & $-1.38 \mathrm{E}-015$ & 100.000 & & & & & & \\
\hline 27 & $-3.83 \mathrm{E}-016$ & $-1.42 \mathrm{E}-015$ & 100.000 & & & & & & \\
\hline
\end{tabular}

Table 5: Principal Component Analysis with un-Rotated Factor solution of Meme Stream Sediment Transformed Geochemical Composition

\begin{tabular}{|c|c|c|c|c|c|c|c|c|}
\hline Elements & $\mathrm{PC} 1$ & $\begin{array}{l}\text { Comp } \\
\text { PC2 }\end{array}$ & PC3 & PC4 & PC5 & PC6 & PC7 & PC8 \\
\hline clr.V & .810 & .177 & .035 & -.277 & .304 & .022 & -.218 & .029 \\
\hline clr.Cr & .619 & -.288 & .182 & .096 & -.101 & .159 & .581 & .282 \\
\hline clr.Ni & -.335 & .104 & -.300 & -.773 & .138 & -.292 & .123 & -.110 \\
\hline clr.Cu & .840 & .280 & .219 & .157 & .065 & .040 & -.261 & -.262 \\
\hline clr.Zn & .588 & -.228 & -.003 & .024 & .191 & .441 & -.134 & .413 \\
\hline clr.Ta & -.775 & .324 & -.207 & .350 & .084 & .028 & .196 & .167 \\
\hline clr.Ga & -.312 & .027 & .331 & .072 & .824 & .232 & -.029 & .080 \\
\hline clr.AS & -.317 & -.548 & .365 & -.324 & -.335 & .126 & -.045 & .462 \\
\hline clr.Pb & .006 & .317 & .882 & .081 & -.047 & .107 & .280 & .113 \\
\hline clr.Rb & .864 & -.266 & -.101 & .128 & .017 & -.054 & -.103 & .255 \\
\hline clr.Sr & .874 & -.196 & .305 & -.117 & -.009 & -.123 & .033 & .033 \\
\hline clr.Hg & .283 & -.829 & -.121 & -.059 & .393 & -.103 & .010 & -.072 \\
\hline clr.Zr & .413 & .318 & .485 & .132 & .490 & -.152 & -.104 & -.050 \\
\hline clr.Nb & -.685 & .294 & -.206 & .376 & .103 & .374 & -.267 & .024 \\
\hline clr.Mo & .138 & .554 & -.250 & -.637 & -.131 & -.045 & -.356 & .179 \\
\hline clr.Fe & .684 & .379 & .397 & .110 & -.231 & -.302 & .078 & -.249 \\
\hline clr.Au & .123 & -.773 & -.162 & .257 & -.199 & -.164 & -.054 & -.391 \\
\hline clr.Ag & -.577 & -.473 & .472 & .317 & -.015 & -.265 & .047 & -.030 \\
\hline clr.Mn & .133 & .067 & .704 & -.042 & -.554 & .253 & -.166 & -.115 \\
\hline clr.Re & .198 & .684 & -.549 & .067 & -.303 & -.144 & .196 & .156 \\
\hline clr.Eu & -.008 & .529 & .060 & .558 & .115 & -.342 & -.368 & .026 \\
\hline clr.Ba & .060 & .321 & .081 & .180 & .152 & -.582 & .661 & .220 \\
\hline clr.Ir & -.455 & -.189 & .501 & -.239 & .141 & .296 & .250 & -.344 \\
\hline clr.In & -.375 & -.299 & .368 & .213 & -.430 & -.379 & -.438 & .239 \\
\hline clr.Ce & .014 & .668 & .267 & -.103 & -.262 & .479 & .254 & -.191 \\
\hline clr.Sb & .386 & -.325 & -.684 & .140 & -.189 & .156 & .266 & -.213 \\
\hline clr.Y & .388 & .013 & -.426 & .590 & -.108 & .415 & .036 & .045 \\
\hline
\end{tabular}

Table 6: Principal Component Analysis with Rotated Factor Solution Of Meme Stream Sediment Transformed Geochemical Composition

\begin{tabular}{|c|c|c|c|c|c|c|c|c|}
\hline Elements & PC1 & $\begin{array}{l}\text { Com } \\
\text { PC2 }\end{array}$ & PC3 & PC4 & PC5 & PC6 & PC7 & PC 8 \\
\hline clr.V & .769 & -.075 & -.381 & .143 & .122 & -.275 & .206 & -.104 \\
\hline clr.Cr & .517 & .138 & .190 & -.420 & .501 & -.110 & -.076 & .441 \\
\hline clr.Ni & -.129 & -.258 & -.362 & -.300 & -.776 & -.177 & -.034 & .057 \\
\hline
\end{tabular}




\begin{tabular}{|c|c|c|c|c|c|c|c|c|}
\hline clr.Cu & .732 & .243 & -.069 & .499 & .253 & -.243 & .035 & -.163 \\
\hline clr.Zn & .432 & -.136 & -.180 & -.242 & .661 & -.057 & .234 & -.161 \\
\hline clr.Ta & -.917 & .021 & -.022 & .134 & -.002 & -.002 & .102 & .274 \\
\hline clr.Ga & -.222 & -.069 & .139 & .012 & -.007 & -.116 & .930 & -.020 \\
\hline clr.AS & -.044 & .058 & .051 & -.690 & -.004 & .696 & .051 & -.107 \\
\hline clr.Pb & .141 & .787 & .114 & .001 & .017 & .227 & .424 & .324 \\
\hline clr.Rb & .729 & -.291 & -.068 & .037 & .531 & -.006 & -.130 & .052 \\
\hline clr.Sr & .935 & .043 & .040 & -.036 & .192 & .008 & -.010 & .098 \\
\hline clr.Hg & .414 & -.665 & .462 & -.307 & .062 & -.051 & .126 & -.114 \\
\hline clr.Zr & .467 & .167 & -.005 & .457 & .019 & -.083 & .561 & .157 \\
\hline clr.Nb & -.866 & .059 & -.068 & .243 & .165 & -.022 & .185 & -.284 \\
\hline clr.Mo & .127 & .041 & -.907 & .047 & -.288 & -.041 & -.112 & -.183 \\
\hline clr.Fe & .683 & .467 & .004 & .457 & -.020 & -.057 & -.183 & .251 \\
\hline clr.Au & .195 & -.383 & .698 & -.057 & .050 & .090 & -.430 & -.239 \\
\hline clr.Ag & -.298 & -.007 & 679 & -.038 & -.176 & .569 & .188 & .111 \\
\hline clr.Mn & .282 & .780 & .099 & -.044 & .042 & .387 & -.074 & -.274 \\
\hline clr.Re & -.146 & .078 & -.596 & .298 & .122 & -.318 & -.508 & .377 \\
\hline clr.Eu & -.138 & .037 & -.121 & .871 & .116 & .156 & .112 & .130 \\
\hline clr.Ba & .029 & .016 & .010 & .178 & -.122 & -.074 & .036 & .969 \\
\hline clr.Ir & -.189 & .382 & .433 & -.395 & -.379 & -.050 & .379 & -.155 \\
\hline clr.In & -.135 & -.003 & .192 & .143 & -.068 & .940 & -.125 & -.092 \\
\hline clr.Ce & -.112 & .859 & -.247 & -.003 & .002 & -.340 & -.004 & -.007 \\
\hline clr.Sb & .108 & -.344 & .171 & -.177 & .328 & -.497 & -.609 & -.047 \\
\hline clr.Y & -.051 & -.054 & .058 & .171 & .785 & -.350 & -.292 & -.076 \\
\hline
\end{tabular}

Table 7: Summary of Important Associations From Factor Analysis Versus Spearman Correlation Of Meme Stream Sediment Transformed Geochemistry

\begin{tabular}{|c|c|c|c|}
\hline Component & $\begin{array}{l}\% \text { of variance ex- } \\
\text { plained }\end{array}$ & Factor Analysis Loadings & Spearman Correlations \\
\hline \multirow[t]{2}{*}{ PC1 } & \multirow[t]{2}{*}{21.73} & (i) $\mathrm{V}-\mathrm{Cr}-\mathrm{Cu}-\mathrm{Zn}-\mathrm{Rb}-\mathrm{Sr}-\mathrm{Hg}-\mathrm{Zr}-\mathrm{Fe}-\mathrm{Au}$ & $\begin{array}{l}\text { Cr vs V (0.42), Cu vs V (0.42), Zn vs V (0.41), Rb vs V (0.50), } \\
\text { Sr vs V (0.55), Hg vs V (0.40), Zr vs V (0.31). }\end{array}$ \\
\hline & & (ii) $\mathrm{Ta}-\mathrm{Nb}$ & $\mathrm{Nb}$ vs $\mathrm{Ta}(0.98)$ \\
\hline $\mathrm{PC} 2$ & 12.39 & $\begin{array}{l}\text { (i) } \mathrm{Pb}-\mathrm{Fe}-\mathrm{Mn}-\mathrm{Ce} \text {. } \\
\text { (ii) } \mathrm{Hg}\end{array}$ & Mn vs $\mathrm{Pb}(0.89), \mathrm{Ce}$ vs $\mathrm{Pb}(0.69)$ \\
\hline \multirow[b]{2}{*}{ PC3 } & \multirow[b]{2}{*}{11.40} & (i) $\mathrm{Au}-\mathrm{Ag}$ & Ag vs $\mathrm{Au}(0.39)$ \\
\hline & & (ii) Mo-Re. & Re vs Mo (0.30) \\
\hline \multirow{2}{*}{ PC4 } & \multirow{2}{*}{10.32} & (i) $\mathrm{Cu}-\mathrm{Zr}-\mathrm{Fe}-\mathrm{Eu}$ & Eu vs $\mathrm{Cu}(0.46)$ \\
\hline & & (ii) $\mathrm{Cr}-\mathrm{As}$ & Cr vs As (0.53) \\
\hline PC5 & 10.20 & (i) $\mathrm{Cr}-\mathrm{Zn}-\mathrm{Rb}-\mathrm{Y}$ & Zn vs $\mathrm{Cr}(0.41), \mathrm{Rb}$ vs $\mathrm{Cr}(0.59)$ \\
\hline PC6 & 10.11 & (i) As-Ag-In & Ag vs As (0.61), In vs As (0.61) \\
\hline \multirow{2}{*}{ PC7 } & \multirow{2}{*}{10.02} & (i) $\mathrm{Ga}-\mathrm{Pb}-\mathrm{Zr}$ & $\mathrm{Pb}$ vs $\mathrm{Ga}(0.78), \mathrm{Zr}$ vs $\mathrm{Ga}(0.41)$ \\
\hline & & (ii) $\mathrm{Au}-\mathrm{Re}-\mathrm{Sb}$. & Sb vs Au (0.52) \\
\hline PC8 & 7.27 & $\mathrm{Cr}-\mathrm{Ba}$ & Ba vs $\mathrm{Cr}(0.50)$ \\
\hline
\end{tabular}

\section{Multivariate analysis}

\subsection{Clusters analysis}


Cluster and dendogram have been used to explore multivariate geochemical patterns associated with ore mineralisation (Obaje et. al., 2015, Lapworth et.al., 2012). The cluster analysis and dendogram were employed to identify multivariate geochemical pattern that could be associated to the inferred mineralization in Lokoja region. The result is presented in (Table 2 and Figure 4). The result revealed two main clusters of variables with six groups: clusters 1 and Cluster 2. Cluster 1 comprise four groups $(1 \mathrm{a}, 1 \mathrm{~b}$ and $1 \mathrm{c})$ while cluster 2 is made up of four groups (2a, 2b and 2c). Group 1a is composed of $\mathrm{Nb}$, Ta Mo, Re, $1 \mathrm{~b}$ is consist of Ni, Ir and 1c is composed of $\mathrm{Hg}$, Au. Group 2a is consist of $\mathrm{Pb}, \mathrm{Ag}, \mathrm{As}, \mathrm{Ga}, \mathrm{Ce}, \mathrm{Fe}$ while $2 \mathrm{~b}$ is made up of Eu, $\mathrm{Zr}, \mathrm{Ba}, \mathrm{Sb}, \mathrm{Y}$ and 2c is composed of $\mathrm{Cu}, \mathrm{Zn}, \mathrm{Rb}, \mathrm{Cr}, \mathrm{Sr}, \mathrm{Mn}, \mathrm{V}$. The cluster elemental grouping suggest a base metal, REEs and gems mineralization.

\subsection{Principal component analysis}

The principal component analysis (PCA) of the stream sediment geochemical composition adopted the steps reported in Ghezelbash et.al. (2019), Obaje et.al., (2015), Sadeghi et al. (2014), Grunsky et.al. (2014) and Carranza (2011) employing: initial, extraction and rotation criteria as given in Table 3. Prior to the application of the PCA, the appropriateness of the data for factor analysis was undertaken using Kaiser-Meyer-Oklin (KMO) measure of sampling adequacy (Obaje et al., 2015). The KMO measure of sampling adequacy gave 0.8 to 0.999 as given in Table 3 which is very good to excellent because the baseline value of 0.6 recommended was well exceeded. Moreover, the Bartlett's test of sphericity yielded statistical significance that further strengthened validity of the factorability of the correlation matrix of the trace element concentrations. Eight principal components were extracted from the factor analysis using eigenvalues greater than 1 and which explained the respective $25.09 \%, 16.86 \%, 14.88 \%, 9.46 \%, 8.28 \%, 7.29 \%, 6.91 \%, 4.67 \%$ of the variance, shown in Table 4 . The cumulative percent of the variance of the initial eigenvalues and extracted sums of squared loading was $93.430 \%$ (Table 4). Furthermore, the scree plot revealed a clear break after the eight component and that further verified the retention of the eight components for further analysis and interpretation using Varimax with Kaiser Normalization and rotated solution. The eight component solution revealed the cumulative percent of the variance as $21.73 \%, 34.12 \%, 45.51 \%, 55.84 \%, 66.04 \%, 76.14 \%, 86.16 \%$ and $93.43 \%$ respectively (Table 4 ). The Varimax with Kaiser Normalization and rotated solution gave cumulative per cent $(93.430 \%)$ of rotation sums of squared loading for component 8 which was consistent with the values revealed in the unrotated and rotated Principal Component Analysis extraction loadings (Tables $4 ; 5$ and 6 )

The steps reported in Lapworth et al. (2012) and Ali (2011) were adopted in which PCA components with respect to their respective Pearson correlation coefficients (PCCs) were employed to interrogate geochemical association and elucidated on ore mineralisation in regions of Asian and Africa. In this study, Spearman correlation coefficients (SCCs) of Meme stream sediments transformed geochemistry reported by Ngozi-Chika et al. (in press) were adopted with respect to their respective PCA components. These were employed to further interrogate the ore element associations towards elucidating on the indicated mineralization in Lokoja and environs; the results are given in Table 6 and Table 7. Spearman correlation coefficients (SCCs) were employed ahead of the PCCs because the latter based its computation with respect to Euclidean distances and its relationship is linear while formal are correlated by a monotonic function and thus gives an enhancement on compositional association patterns towards elucidating on the presence of mineralisation in the region which is beyond a linear representation of elemental associations as usually depicted by PCCs. The PCA analysis showed that Factor 1 explains $21.73 \%$ of the data variability and is dominated by positive loadings for these ore elements $\mathrm{V}-\mathrm{Cr}-\mathrm{Cu}-\mathrm{Zn}-\mathrm{Rb}-\mathrm{Sr}-\mathrm{Hg}-\mathrm{Zr}-\mathrm{Fe}-\mathrm{Au}$. Dominant negative loadings consist of Ta-Nb. The Spearman correlation coefficients of the positive loadings transition metals gave $\mathrm{Cr}$ vs $\mathrm{V}(0.42)$, Cu vs $\mathrm{V}$ (0.42), Zn vs V (0.41), Rb vs V (0.50), Sr vs V (0.55), Hg vs V (0.40), Zr vs V (0.31) indicating weak to moderate correlation coefficient while the dominant negative loadings gave $\mathrm{Nb}$ vs Ta $(0.98)$ revealing very high correlation coefficient. Factor 2 explains $12.39 \%$ of the data variability and is dominated by positive loadings of transition metals such as $\mathrm{Pb}-\mathrm{Fe}-\mathrm{Mn}$-Ce while the dominant negative loading is $\mathrm{Hg}$. The Spearman correlation of the positive loadings transition metals gave $\mathrm{Mn}$ vs $\mathrm{Pb}(0.89), \mathrm{Ce} v \mathrm{~Pb}(0.69)$ indicating moderate to high correlation coefficients. Factor 3 explains $11.40 \%$ of variability and characterized by positive loadings of $\mathrm{Au}$-Ag while negative loadings consist of Mo-Re. The Spearman correlation of the positive loadings gave Ag vs Au (0.39) and negative loadings gave Re vs Mo (0.30) with weak correlation coefficients for both the positive and negative loadings. Factor 4 explains $10.32 \%$ of variance. The positive loadings are dominated by $\mathrm{Cu}-\mathrm{Zr}$-Fe-Eu while the negative loadings are characterized by $\mathrm{Cr}$-As. The Spearman correlation of the positive loadings gave $\mathrm{Eu}$ vs $\mathrm{Cu}(0.46)$ indicating weak correlation coefficient while the while negative loadings gave $\mathrm{Cr}$ vs $\mathrm{As}(0.53)$ indicating moderate correlation coefficient. Factor 5 explains $10.20 \%$ of variability. The positive loadings are dominated by $\mathrm{Cr}-\mathrm{Zn}-\mathrm{Rb}-\mathrm{Y}$ while negative loadings are negligible; the Spearman correlation of the positive loadings of ore element gave $\mathrm{Zn} v \mathrm{vs} \mathrm{Cr}(0.41), \mathrm{Rb}$ vs $\mathrm{Cr}(0.59)$ showing weak to moderate correlation coefficients while negative loadings are negligible. Factor 6 explains $10.11 \%$ of variance. The positive loadings consists of As-Ag-In while the values of the negative loadings are also negligible. The Spearman correlation coefficients of the positive loadings gave Ag vs As (0.61), In vs As (0.61) showing moderate correlation coefficients. Factor 7 explains $10.02 \%$ of data variability. The positive loadings are composed of $\mathrm{Ga}-\mathrm{Pb}-\mathrm{Zr}$ while negative components consist of $\mathrm{Au}-\mathrm{Re}-\mathrm{Sb}$. The Spearman coefficients of the positive loadings gave $\mathrm{Pb}$ vs $\mathrm{Ga}(0.78), \mathrm{Zr}$ vs Ga (0.41) indicating high to weak correlation coefficients while negative loadings gave $\mathrm{Sb}$ vs $\mathrm{Au}$ (0.52) indicating moderate correlation coefficients. Factor 8 explains $7.27 \%$ of the data variance and positive loadings consist of $\mathrm{Cr}-\mathrm{Ba}$ while the values of negative loadings are negligible. The Spearman coefficients of the positive loadings gave $\mathrm{Ba} v \mathrm{vs}(0.50)$ showing moderate correlation coefficients while the values of negative loadings are negligible.

\section{Conclusion}

The multivariate study of Meme stream sediment compositional data of Lokoja and environs was carried out employing centered log-ratio (clr) transformed geochemical composition data to elucidate on the indicated mineralization in the region. The positive and negative loadings of PC indicated the presences of oxides, sulphides, REEs and gems mineralization in the Lokoja and environs. Respective PCA components were further interrogated using SCCs as shown in Table 7 indicated very high to moderate correlation coefficients between Sr and $\mathrm{V}$ (0.55), $\mathrm{Mn}$ and $\mathrm{Pb}(0.89), \mathrm{Nb}$ vs $\mathrm{Ta}(0.98), \mathrm{Ag}$ and $\mathrm{Au}(0.39), \mathrm{Re}$ and $\mathrm{Mo}(0.30), \mathrm{Eu}$ and $\mathrm{Cu}(0.46), \mathrm{Rb}$ and $\mathrm{Cr}(0.59), \mathrm{In}$ and $\mathrm{As}$ (0.64), $\mathrm{Pb}$ and $\mathrm{Ga}(0.78), \mathrm{Sb}$ and $\mathrm{Au}(0.52), \mathrm{Ba}$ and $\mathrm{Cr}(0.50)$. These well-developed elemental association suggests that these ore elements are either indicator of their own mineralization or are suitable pathfinders to pertinent minerals in Lokoja region. In addition, negative correlation between $\mathrm{Fe}$ and other ore elements indicated that the $\mathrm{Fe}$ is from both proximal and dextral sources probably due to many $\mathrm{Fe}$ formations and mineralization (goethite, haematite \pm siderite - bearing sedimentary ironstone formations occurred scattered within the region). Furthermore, the high correlation coefficients between $\mathrm{Mn}$ and $\mathrm{Ta}(0.77), \mathrm{Mn}$ and $\mathrm{Nb}(0.78)$ indicated that these ore elements were 
from the proximal sources because $\mathrm{Mn}, \mathrm{Fe}$, Ta and $\mathrm{Nb}$ are reliable pathfinders to pertinent oxides mineralisation in the region (NgoziChika et al., in press). Some of the proximal mineralisation in the region include beryl, topaz, columbite, quartzofeldspathic and quartz veins with anomalous concentration of $\mathrm{Au}$ as well as industrial minerals. The industrial minerals occurred as a by-product of the mineralisation process which is artisanally mined in places for industrial purposes.

\section{References}

[1] Ali, L. (2011). Gold and base metal exploration studies based on mineralogical and geochemical characterization of stream sediments from North Pakistan. Unpublished Ph.D. Thesis, Camborne School of Mine, University of Exeter, UK.

[2] Aitchison, J. (1986). The Statistical Analysis of Compositional Data. Chapman and Hall, London. https://doi.org/10.1007/978-94-009-4109-0.

[3] Carranza, E.J.M., (2011). Analysis and mapping of geochemical anomalies using logratio-transformed stream sediment data with censored values. Journal of Geochemical Exploration 110, 167 - 185. https://doi.org/10.1016/j.gexplo.2011.05.007.

[4] Filzmoser, P. Hron, K. \& Reimann, C. (2009). Univariate statistical analysis of environmental (compositional) data - Problems and Possibilities. Science of the Total Environment 407: 6100-6108. https://doi.org/10.1016/j.scitotenv.2009.08.008.

[5] Filzmoser, P., Hron, K., Reimann, C. (2009). Principal component analysis for compositional data with outliers. Environmetrics 20: 621-632 https://doi.org/10.1002/env.966.

[6] Ghezelbash, R., Maghsoudi, A., Carranza, E. J. M. (2019). Mapping of single- and multi-element geochemical indicators based on catchment basin analysis: Application of fractal method and unsupervised clustering models, Jour of Geoch. Explorat. Retrived from https://doi.org/10.1016/j.gexplo.2019.01.017.

[7] Grunsky, E.C., (2010). The interpretation of geochemical survey data. Geochemistry. Exploration, Environment and Analysis 10, 27-74. https://doi.org/10.1144/1467-7873/09-210.

[8] Haruna, I.V (2017). Review of the Basement Geology and Mineral Belts of Nigeria. Journal of Applied Geology and Geophysics (IOSR-JAGG), Vol. 5, Issue 1 Ver. I, $37-45$

[9] Lapworth, D.J., Knights, K.V., Key, R.M., Johnson, C.C, Ayoade, E., Adekanmi, M.A., Arisekola, T.M., Okunlola, O.A., Backman, B., Eklund, M., Everett, P.A., Lister, T.R., Ridgway, J., Watts, M.J., Kemp, S.J. \& Pitfield, P.E.J. (2012). Geochemical mapping using stream sediments in westcentral Nigeria: Implications for environmental studies and mineral exploration in West Africa. Applied Geochemistry, 27(6) : 1035-1052 https://doi.org/10.1016/j.apgeochem.2012.02.023.

[10] Ngozi-Chika, C.S., Ibrahim, O.I. and Ugwueze, C.U. In Press. Stream sediment geochemical survey of Meme river drainage basin, Lokoja, NorthCentral, Nigeria: Implications for Mineral Prospecting. Completed and submitted Manuscript.

[11] Obaje, N.G. (2009). Geology and Mineral Resources of Nigeria. Springer Dordrecht Hudelberg London New York, 221 pp. https://doi.org/10.1007/978-3-540-92685-6.

[12] Obaje, S.O., Akpoborie, I.A., Ugbe, F.C., and Onugba, A. (2015). "Rare Earth and Trace Elements Distribution in Sediments from River Gora in Minna Area, North- Central Nigeria: Implication for Provenance", Earth Science Research, 3(1), 28-33. https://doi.org/10.5539/esr.v4n1p103.

[13] Omada, J.I., Kolawole, M.S. and Odoma, A.N. (2015). Field and petrochemical studies of pegmatites in parts of Lokoja, Central Nigeria. Journal of African Earth Sciences. 101, 266-273 https://doi.org/10.1016/j.jafrearsci.2014.09.020.

[14] Reimann C, Filzmoser P, Garrett RG, Dutter R. (2008). Statistical data analysis explained. Applied Environmental Statistics with R. Chichester: Wiley, 343 pp. https://doi.org/10.1002/9780470987605.

[15] Reimann, C., Filzmoser, P., Fabian, K., Hron, K., Birke, M., Demetriades, A., Dinelli, E., Ladenberger, A., The GEMAS Project Team (2012). The concept of compositional data analysis in practice - Total major element concentrations in agricultural and grazing land soils of Europe. Science of the Total Environment 426:196-210. https://doi.org/10.1016/j.scitotenv.2012.02.032.

[16] Sadeghi, M., Billay, A., John, E. and Carranza, M. (2014) Analysis and mapping of soil geochemical anomalies: implications for bedrock mapping and gold exploration in Giyani area, South Africa. Jour. of Geochemical Exploration, https://doi.org/10.1016/j.gexplo.2014.11.018. 